\title{
An Axiomatic Characterization of Ensconcement-Based Contraction
}

\author{
EDUARDO FERMÉ, Departamento de Matemática e Engenharias - Universidade \\ da Madeira Campus Universitário da Penteada, 9000-390 Funchal Portugal. \\ E-mail:ferme@uma.pt
}

MARTÍN KREVNERIS, Gestion-Digital, Buenos Aires, Argentina. E-mail:mkrev@gestion-digital.com.ar

\begin{abstract}
MAURÍCIO REIS, Departamento de Matemática e Engenharias - Universidade da Madeira Campus Universitário da Penteada, 9000-390 Funchal Portugal. E-mail:m_reis@uma.pt
\end{abstract}

\begin{abstract}
In this article, we propose an axiomatic characterization for ensconcement-based contraction functions, belief base functions proposed by Williams. We relate this function with other kinds of base contraction functions.
\end{abstract}

Keywords: Logic of theory change, belief bases, base contraction, ensconcement.

\section{Introduction}

The logic of theory change became a major subject in philosophical logic and artificial intelligence in the middle of the 1980's. The most important model, now known as the AGM model of belief change, was proposed by Alchourrón, Gärdenfors and Makinson in [1]. The AGM model is a formal framework to characterize the dynamics and state of belief of a rational agent. The beliefs of an agent are represented by a set of sentences closed under logical consequence. The AGM model has acquired the status of a standard model, and has been characterized in at least five different equivalent ways: Postulates [1, 16], partial meet functions [1,3], epistemic entrenchment [17, 18], safe/kernel contraction [4, 23] and Grove's sphere-systems [19].

One of the most important variants of the AGM model is to represent the beliefs of an agent by a belief base, a set of sentences that is not (necessarily) closed under logical consequence.

Partial meet contraction and kernel contraction have been characterized for belief bases [19, 20,23]. Mary-Anne Williams proposed a model of contraction based on an 'ensconcement' [35, 37, 38], which is closely related to epistemic entrenchment. In this article, we provide an axiomatic characterization of ensconcement-based contraction functions.

\section{Belief bases}

In the AGM model, an individual's belief state is represented by a belief set-a set of sentences that is closed under logical consequences-and changes in belief are represented by operations on such sets. Several formal models have been proposed for the contraction of belief sets. One alternative 
framework for the representation of belief states is by belief bases-sets of sentences that are not (necessarily) closed under logical consequence.

Several authors analysed the use of belief bases [6, 8, 15, 20, 22, 24, 28, 31, 34]. Among the advantages of the use of that approach instead of using belief sets we can mention:

1. Belief bases have more expressive power: on the one hand, belief bases allow to distinguish between basic beliefs and those which were inferred from them. For instance, if an agent believes 'Paris is the Capital of France', he also believes that 'Paris is the Capital of France or the Moon is a piece of cheese'. However, the agent believes in the latter belief 'just because' he believes in the former. The 'just because-sentences' were studied by Fermé [9], Furhmann [15] and Rott [31]. On the other hand, $A=\{\alpha, \beta\}$ and $B=\{\alpha \wedge \beta\}$ have the same logical consequences, and consequently, generate the same belief set. However, the difference between $A$ and $B$ is not just a 'notational bondage' that should be straightened out by some process of 'articulation' (Belnap [5], cited by Rott in [31]).

2. Belief bases allow to represent resource bounded agents: ideal rational agents can have infinite inferential power or infinite representation of belief. However, when we want to represent 'real' agents, we need to think of agents with bounded resources. The use of belief bases is one step in this direction. For belief change in resource bounded agents see [34].

3. Using belief bases it is possible to distinguish between different inconsistent belief states [20]: agents are, sometimes, inconsistent. However, this does not mean that all the inconsistent agents have the same beliefs. The belief sets just recognize one inconsistent belief set, that contains all the sentences of the language (for a work on local inconsistencies in agents see [27]).

4. Belief sets are very large entities: for practical applications, we have to work on belief bases, because the implications of any belief base may be an infinite (or an extremely large) set of propositions. For instance, if $\alpha$ is in the belief set then so are both $\alpha \vee \beta$ and $\alpha \vee \neg \beta$ for any $\beta$ in the language. Even if we restrict the belief sets to the sets with finite axiomatization, performing changes is exponentially large. For example, if a belief set is simply $\operatorname{Cn}(\alpha)$, to contract by $\alpha$ implies to decide between (excluding one or both of) $\alpha \vee \beta$ and $\alpha \vee \neg \beta$ for any $\beta$ in the language. For computer implementations, the use of belief bases is more suitable. For an overview of the computational cost of performing changes in belief bases, see [29].

\section{Contraction functions for belief bases}

Formal preliminaries: We will assume a language $\mathcal{L}$ that is closed under truth-functional operations and a consequence operator $C n$ for $\mathcal{L}$. $C n$ satisfies the standard Tarskian properties, namely inclusion $(A \subseteq C n(A)$ ), monotony (if $A \subseteq B$, then $C n(A) \subseteq C n(B))$ and iteration $(C n(A)=C n(C n(A))$ ). It is supraclassical and compact, and satisfies deduction (if $\beta \in C n(A \cup\{\alpha\}$ ), then $(\alpha \rightarrow \beta) \in C n(A)$ ). $A \vdash \alpha$ will be used as an alternative notation for $\alpha \in C n(A), \vdash \alpha$ for $\alpha \in C n(\emptyset)$ and $\operatorname{Cn}(\alpha)$ for $\operatorname{Cn}(\{\alpha\})$. Upper-case letters denote subsets of $\mathcal{L}$. Lower-case Greek letters denote elements of $\mathcal{L}$. Base expansion is simply a set union, i.e. $A+\alpha=A \cup\{\alpha\}$. A contraction of $A$ with respect to $\alpha$ involves removal of a set of sentences from $A$ so that $\alpha$ is no longer implied.

\subsection{Partial meet contraction}

We can construct a base contraction function using remainder sets, i.e. maximal subsets of $A$ that fail 
to imply $\alpha$ :

DEFINITION 1 [2]

Let $A$ be a belief base and $\alpha$ a sentence. The set $A \perp \alpha$ (A remainder $\alpha)$ is the set of sets such that $B \in A \perp \alpha$ if and only if:

- $B \subseteq A$

- $B \nvdash \alpha$

- There is no set $B^{\prime}$ such that $B \subset B^{\prime} \subseteq A$ and $B^{\prime} \nvdash \alpha$

DEFINITION $2[1,3]$

Let $A$ be a belief base. A selection function for $A$ is a function $\gamma$ such that for all sentences $\alpha$ :

1. If $A \perp \alpha$ is non-empty, then $\gamma(A \perp \alpha)$ is a non-empty subset of $A \perp \alpha$.

2. If $A \perp \alpha$ is empty, then $\gamma(A \perp \alpha)=\{A\}$.

DEFINITION 3 [1, 20]

The partial meet base contraction operator on $A$ based on a selection function $\gamma$ is the operator $-\gamma$ such that for all sentences $\alpha$ :

$$
A-\gamma \alpha=\bigcap \gamma(A \perp \alpha)
$$

Hansson characterized partial meet base contraction in terms of postulates:

THEOREM 4 [20]

Let $A$ be a belief base. An operator - on $A$ is a partial meet contraction function for $A$ if and only if - satisfies

Success If $\nvdash \alpha$, then $A-\alpha \nvdash \alpha$.

Inclusion $A-\alpha \subseteq A$.

Relevance If $\beta \in A$ and $\beta \notin A-\alpha$ then there is some set $A^{\prime}$ such that $A-\alpha \subseteq A^{\prime} \subseteq A$ and $\alpha \notin C n\left(A^{\prime}\right)$ but $\alpha \in \operatorname{Cn}\left(A^{\prime} \cup\{\beta\}\right)$.

Uniformity If it holds for all subsets $A^{\prime}$ of $A$ that $\alpha \in C n\left(A^{\prime}\right)$ if and only if $\beta \in \operatorname{Cn}\left(A^{\prime}\right)$, then $A-\alpha=A-\beta$.

Partial meet base contraction also satisfies other interesting properties as we can see in the following observation:

OBSERVATION 5 [25]

Let $A$ be a belief base and - an operator on $A$. Then:

1. If - satisfies inclusion and relevance, then it satisfies vacuity (If $A \nvdash \alpha$, then $A \subseteq A-\alpha$ ) and failure (If $\vdash \alpha$, then $A-\alpha=A$ ).

2. If - satisfies uniformity, then it satisfies extensionality (If $\vdash \alpha \leftrightarrow \beta$, then $A-\alpha=A-\beta$ ).

\subsection{Kernel contraction}

In [23] Hansson introduced Kernel Contraction, a generalization of Safe Contraction [4]. It is based on a selection among the sentences of a set $A$ that contribute effectively to imply $\alpha$; and on how to 
use this selection in contracting by $\alpha$. Formally:

DEFINITION 6 [23]

Let $A$ be a set in $\mathcal{L}$ and $\alpha$ a sentence. Then $A \Perp \alpha$ is the set such that $B \in A \Perp \alpha$ if and only if:

$$
\left\{\begin{array}{l}
B \subseteq A \\
B \vdash \alpha \\
\text { If } B^{\prime} \subset B \text { then } B^{\prime} \nvdash \alpha
\end{array}\right.
$$

$A \Perp \alpha$ is called the kernel set of $A$ with respect to $\alpha$ and its elements are the $\alpha$-kernels of $A$.

DEFINITION 7 [23]

Let $A$ be a set of sentences. Let $A \Perp \alpha$ be the kernel set of $A$ with respect to $\alpha$. An incision function $\sigma$ for $A$ is a function such that for all sentences $\alpha$ :

$$
\left\{\begin{array}{l}
\sigma(A \Perp \alpha) \subseteq \bigcup(A \Perp \alpha) \\
\text { If } \emptyset \neq B \in A \Perp \alpha, \text { then } B \cap \sigma(A \Perp \alpha) \neq \emptyset
\end{array}\right.
$$

DEFINITION 8 [23]

Let $A$ be a set of sentences and $\sigma$ an incision function for $A$. The kernel contraction $-{ }_{\sigma}$ for $A$ is defined as:

$$
A-{ }_{\sigma} \alpha=A \backslash \sigma(A \Perp \alpha) \text {. }
$$

An operator - for a set $A$ is a kernel contraction if and only if there is an incision function $\sigma$ for $A$ such that $A-\alpha=A-{ }_{\sigma} \alpha$ for all sentences $\alpha$.

Hansson also provided an axiomatic characterization for kernel contraction:

THEOREM 9 [23]

The operator - for a set of sentences $A$ is a kernel contraction if and only if it satisfies success, inclusion, uniformity and

Core-retainment If $\beta \in A$ and $\beta \notin A-\alpha$ then there is some set $A^{\prime}$ such that $A^{\prime} \subseteq A$ and $\alpha \notin C n\left(A^{\prime}\right)$ but $\alpha \in C n\left(A^{\prime} \cup\{\beta\}\right)$.

Since core-retainment is weaker than relevance, it follows that, for belief bases, all partial meet contractions are kernel contractions. There exist two more conservative types of kernel contraction:

A kernel contraction is smooth if and only if for all subsets $A^{\prime}$ of $A$ : if $A^{\prime} \vdash \beta$ and $\beta \in \sigma(A \Perp \alpha)$ then $A^{\prime} \cap \sigma(A \Perp \alpha) \neq \emptyset[23]$.

A kernel contraction is relevant if and only if for all $\beta \in \sigma(B \Perp \alpha)$, there is an $X$ such that $(B \backslash \sigma(B \Perp \alpha)) \subseteq X \subseteq B, X \nvdash \alpha$ and $X \cup\{\beta\} \vdash \alpha[10]$.

THEOREM 9 (cont.)

- [23] - is smooth if and only if it also satisfies:

Relative Closure $A \cap C n(A-\alpha) \subseteq A-\alpha$.

- [10] - is relevant if and only if it also satisfies relevance. 


\subsection{Ensconcement}

Mary-Anne Williams [35, 37, 38] defines an ensconcement relation on a belief base A as a transitive and connected relation $\preceq$ that satisfies the following three conditions: ${ }^{1}$

( $\preceq \mathbf{1})$ If $\beta \in A \backslash C n(\emptyset)$, then $\{\alpha \in A: \beta \prec \alpha\} \nvdash \beta$.

( 22$)$ If $\forall \alpha$ and $\vdash \beta$, then $\alpha \prec \beta$, for all $\alpha, \beta \in A$.

( $\preceq 3)$ If $\vdash \alpha$ and $\vdash \beta$, then $\alpha \preceq \beta$, for all $\alpha, \beta \in A$.

( $\preceq 1)$ says that the formulae that are strictly more ensconced than $\alpha$ do not (even conjointly) imply $\alpha$. Conditions $(\preceq 2)$ and $(\preceq 3)$ say that tautologies are the most ensconced formulae. Given an ensconcement relation, a cut operator is defined by:

$$
\operatorname{cut}_{A}(\alpha)=\{\beta \in A:\{\gamma \in A: \beta \preceq \gamma\} \not \forall \alpha\}
$$

LEMMA 10 [36]

If $\alpha \in A, \operatorname{cut}_{A}(\alpha)=\{\beta \in A: \alpha \prec \beta\}$.

The previous lemma says that when $\alpha$ is an explicit belief, its cut is the subset of $A$ such that its members are strictly more ensconced than $\alpha$. Other interesting properties of $c u t$ are:

\section{LEMMA 11}

(a) If $\vdash \alpha$, then $\operatorname{cut}_{A}(\alpha)=\emptyset$.

(b) If $\forall \alpha$, cut $_{A}(\alpha) \nvdash \alpha$.

(c) If $A \nvdash \not \alpha$, cut $_{A}(\alpha)=A$.

(d) If $\beta \vdash \alpha$, then $\operatorname{cut}_{A}(\alpha) \subseteq \operatorname{cut}_{A}(\beta)$.

(e) If $\vdash \alpha \leftrightarrow \beta$, then $\operatorname{cut}_{A}(\alpha)=\operatorname{cut}_{A}(\beta)$.

(f) If $\alpha \preceq \beta$, then $\operatorname{cut}_{A}(\beta) \subseteq \operatorname{cut}_{A}(\alpha)$.

(g) If $\alpha \prec \beta$, then $\operatorname{cut}_{A}(\alpha) \vdash \beta$ and $\operatorname{cut}_{A}(\beta) \nvdash \alpha$.

(h) If $\alpha \prec \beta$, then $\operatorname{cut}_{A}(\alpha \wedge \beta)=\operatorname{cut}_{A}(\alpha)$.

(i) If $\beta=\preceq \alpha$, then $\operatorname{cut}_{A}(\alpha \wedge \beta)=\operatorname{cut}_{A}(\alpha)=\operatorname{cut}_{A}(\beta)$.

(j) If $\operatorname{cut}_{A}(\alpha) \vdash \beta$, then $\operatorname{cut}_{A}(\alpha \wedge \beta)=\operatorname{cut}_{A}(\alpha)$.

(k) If $\operatorname{cut}_{A}(\alpha) \nvdash \beta$, then $\operatorname{cut}_{A}(\alpha \wedge \beta)=\operatorname{cut}_{A}(\beta)$.

We can define a base contraction operator - using the cut operator:

\section{DEFINITION 12}

Let $A$ be a belief base and $\preceq$ an ensconcement relation. Then - is an Ensconcement-Based Contraction Function if and only if: ${ }^{2}$

$$
\beta \in A-\alpha \text { if and only if } \beta \in A \text { and either (i) } \alpha \in C n(\emptyset) \text { or (ii) } c u t_{A}(\alpha) \vdash \alpha \vee \beta
$$

Related Works: Besides epistemic entrenchment, we found in the belief change literature, other approaches related with ensconcement. On the one hand, in quantitative formalism, Dubois and Prade [7, Sec. 2.5] remarked that a possibility or a necessity measure is no more than a preorder on logical

\footnotetext{
${ }^{1} \alpha \prec \beta$ means $\alpha \preceq \beta$ and $\beta \preceq \alpha . \alpha=\preceq \beta$ means $\alpha \preceq \beta$ and $\beta \preceq \alpha$.

${ }^{2}$ Note that condition (ii) requires that cut $_{A}(\alpha) \vdash \alpha \vee \beta$ instead of the more intuitive condition cut $_{A}(\alpha) \vdash \beta$. Mary-Anne Williams calls a contraction based on this alternative condition 'Brutal Theory Base Contraction'. Brutal Theory Base Contraction is closely related to Severe Withdrawal [30], whereas Ensconcement-Based Contraction is closely related to AGM contraction.
} 


\section{Axiomatic Characterization of Ensconcement-Based Contraction}

sentences. Spohn [33] defined a model of ordinal conditional functions where the order is determined by the level of implausibility of their negations. On the other hand, in qualitative formalism, Rott [30] presented the E-bases, a preference relation that provides a canonical representation of an epistemic entrenchment and a belief base. Williams [38] pointed out that the E-bases approach is equivalent to the ensconcement approach. For a discussion about quantitative versus qualitative formalism see $[14$, Sec. 1$]$.

\section{Axioms for Ensconcement-Based Contraction Functions}

In this section, we are going to investigate the postulates that characterize the Ensconcement-Based Contraction Functions. The following postulates are well-known in the belief revision literature: Success, Inclusion,

Vacuity If $A \nvdash \alpha$, then $A \subseteq A-\alpha$,

Extensionality If $\vdash \alpha \leftrightarrow \beta$, then $A-\alpha=A-\beta$,

Conjunctive Factoring $A-\alpha \wedge \beta=\left\{\begin{array}{l}A-\alpha \text { or } \\ A-\beta \text { or } \\ A-\alpha \cap A-\beta\end{array}\right.$

The question that arises here is what is the postulate that characterizes the notion of 'minimal change' in ensconcement. We propose the following postulate:

Disjunctive Elimination If $\beta \in A$ and $\beta \notin A-\alpha$ then $A-\alpha \nvdash \alpha \vee \beta$.

It is important to note the relation between disjunctive elimination and other postulates:

\section{OBSERVATION 13}

Let $A$ be a belief base and - an operator on $A$ that satisfies disjunctive elimination. Then - satisfies:

Relative Closure $A \cap \operatorname{Cn}(A-\alpha) \subseteq A-\alpha$.

If - also satisfies inclusion then it satisfies:

Failure If $\vdash \alpha$ then $A-\alpha=A$.

By means of Disjunctive Elimination we can characterize Ensconcement-Based Contraction Functions:

\section{THEOREM 14}

Let $A$ be a belief base. An operator - of $A$ is an ensconcement-based contraction on $A$ if and only if it satisfies success, inclusion, vacuity, extensionality, disjunctive elimination and conjunctive factoring.

Due to Observation 13 every ensconcement-based contraction function satisfies failure and relative closure.

OBSERVATION 15

An ensconcement-based contraction function - on $A$ does not, in general, satisfy uniformity.

\section{Ensconcement and minimal change}

As we showed, ensconcement-based contraction satisfies vacuity, failure and relative closure. Vacuity guarantees the minimal change (i.e. to do nothing) when the sentence to be contracted is not implied by the original belief base. Failure [11], means that when instructed to do the impossible (to contract 
a tautology), the minimal change is to do nothing. Relative closure was introduced in [23] and ensures that original beliefs that are implied by the contracted set are not gratuitously removed.

In the previous section, we mentioned that disjunctive elimination is the postulate that characterizes the notion of minimal change in ensconcement-based contraction. In its original formulation (where the precondition is the same as in core-retainment and relevance), the consequence $A-\alpha \nvdash \alpha \vee \beta$ appears as a negative condition. However, if we reformulate the postulate as

$$
\text { If } \beta \in A \text { and } A-\alpha \vdash \alpha \vee \beta \text {, then } \beta \in A-\alpha \text {, }
$$

we can see the postulate as a condition for a sentence $\beta$ 'to survive' the contraction process. If we reformulate relative closure as

$$
\text { If } \beta \in A \text { and } A-\alpha \vdash \beta \text {, then } \beta \in A-\alpha \text {, }
$$

we can see relative closure as a weaker version of disjunctive elimination, that requires that the original beliefs that are implied by the contracted set should be elements of the contracted set.

In belief bases, disjunctive elimination is a weaker condition than relevance:

OBSERVATION 16

Let A be a belief base. Then relevance implies disjunctive elimination.

In [31], Rott points out that ensconcement-based contraction gratuitously loses independent beliefs with a low priority in the belief base, as we see in the following example:

\section{EXAMPLE 17}

Let $\alpha$ and $\beta$ be logically independent sentences and let $A=\{\alpha, \beta, \beta \rightarrow \delta\}$. Let $\preceq$ be an ensconcement on $A$ such that $\beta \prec \alpha \prec \beta \rightarrow \delta$. Let - be the ensconcement-based contraction for $A$ based on $\preceq$. Hence $A-\alpha=\{\beta \rightarrow \delta\}$, and $\beta$ was lost.

This happens because, in general, core-retainment fails for ensconcement-based contractions. We note also that even if an ensconcement-based contraction satisfies core-retainment it may not satisfy relevance:

OBSERVATION 18

Let $\mathrm{A}$ be a belief base and - an operator on $A$. Then core-retainment and disjunctive elimination do not imply relevance.

The difference between relevance and disjunctive elimination disappears if $\mathrm{A}$ is a belief set.

OBSERVATION 19

Let $A$ be a belief set and - and operator on $A$ that satisfies inclusion and vacuity. Then disjunctive elimination implies relevance.

\section{Maps between different base contraction functions}

Mary-Anne Williams demonstrated that an ensconcement-based contraction can be related to an AGM contraction function:

OBSERVATION 20 [36]

For every ensconcement-based contraction - on a belief base $A$ there is an AGM contraction $\div$ on the corresponding belief set $\operatorname{Cn}(A)$ (that satisfies the basic and supplementary postulates for belief 


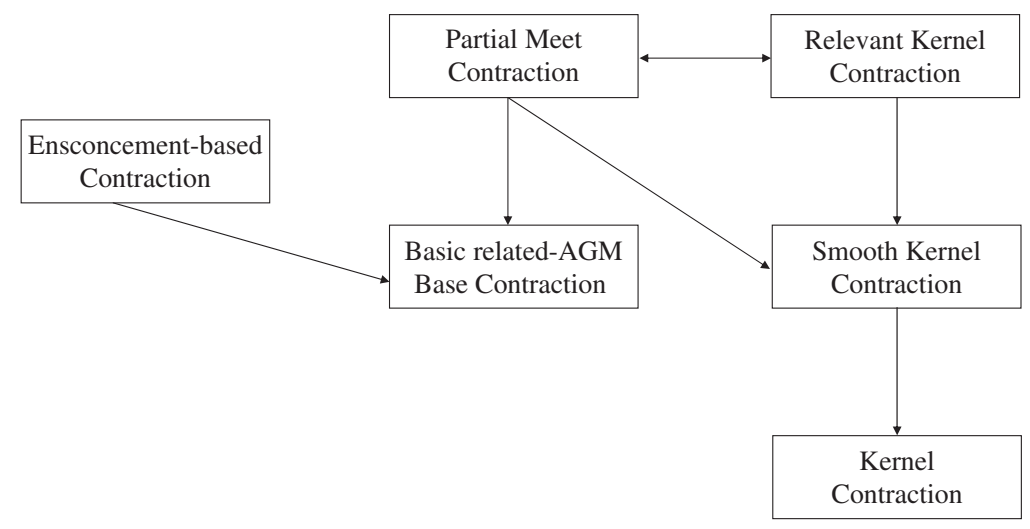

FIGURE 1. Map among different kinds of base contraction functions

set contraction) such that for all sentences $\alpha$ :

$$
A-\alpha=(\operatorname{Cn}(A) \div \alpha) \cap A
$$

We can generalize this result just for the basic postulates:

OBSERVATION 21

Let $A$ be a belief base. Let - be an operator on $A$. Then - satisfies success, inclusion, vacuity, extensionality and disjunctive elimination iff there exists some basic AGM contraction $\div$ for $\mathrm{Cn}(A)$ such that

$$
A-\alpha=(\operatorname{Cn}(A) \div \alpha) \cap A
$$

We will call - a basic related-AGM base contraction.

Due to Observations 5, 13 and 16, it follows that every partial meet contraction is a basic relatedAGM base contraction. With this remark we can construct the actual map of the base contraction functions as shown in Figure 1.

The following example illustrates the differences in the outcomes of the various contraction operators considered: ${ }^{3}$

EXAMPLE 22

Let $\alpha, \beta$ and $\delta$ be logically independent sentences and let

$A=\{\alpha, \alpha \vee \beta, \alpha \leftrightarrow \beta, \delta\}$.

Using the definitions of $\Perp$ and $\perp$ we obtain:

$A \Perp(\alpha \wedge \beta)=\{\{\alpha, \alpha \leftrightarrow \beta\},\{\alpha \vee \beta, \alpha \leftrightarrow \beta\}\}$

and

$A \perp(\alpha \wedge \beta)=\{\{\alpha, \alpha \vee \beta, \delta\},\{\alpha \leftrightarrow \beta, \delta\}\}$

We can define an ensconcement $\preceq$, incision functions $\sigma_{1}, \sigma_{2}$ (smooth) and $\sigma_{3}$ (relevant), and a selection function $\gamma$ on $A$ :

\footnotetext{
${ }^{3}$ This example is a modified version of $[10,26]$.
} 


$$
\begin{aligned}
& \prec: \delta \prec \alpha \leftrightarrow \beta \prec \alpha \prec \alpha \vee \beta, \text { then } \text { cut }_{A}(\alpha \wedge \beta)=\{\alpha, \alpha \vee \beta\} . \\
& \sigma_{1}(A \Perp(\alpha \wedge \beta))=\{\alpha \vee \beta, \alpha \leftrightarrow \beta\} \\
& \sigma_{2}(A \Perp(\alpha \wedge \beta))=\{\alpha, \alpha \leftrightarrow \beta\} \\
& \sigma_{3}(A \Perp(\alpha \wedge \beta))=\{\alpha \leftrightarrow \beta\} \\
& \gamma(A \perp \alpha)=\{\{\alpha, \alpha \vee \beta, \delta\}\}
\end{aligned}
$$

We can define different contraction functions:

$A-\prec(\alpha \wedge \beta)=\{\alpha, \alpha \vee \beta\}$

$A-\sigma_{1}(\alpha \wedge \beta)=\{\alpha, \delta\}$

$A-\sigma_{2}(\alpha \wedge \beta)=\{\alpha \vee \beta, \delta\}$

$A-\sigma_{3}(\alpha \wedge \beta)=\{\alpha, \alpha \vee \beta, \delta\}$

$A-\gamma(\alpha \wedge \beta)=\{\alpha, \alpha \vee \beta, \delta\}$

- $-_{\prec}$ is an ensconcement-based contraction but not a kernel contraction, neither a partial meet contraction (since $\delta \notin A-\prec(\alpha \wedge \beta)$, this violates core-retainment and relevance).

- $-\sigma_{\sigma_{1}}$ is a kernel contraction but not a smooth kernel contraction (since $\alpha \vee \beta \notin A-{ }_{\sigma_{1}}(\alpha \wedge \beta)$, this violates relative closure).

- $-\sigma_{2}$ is a smooth kernel contraction but not a relevant kernel contraction ( $\operatorname{since} \alpha \notin A-\sigma_{2}(\alpha \wedge \beta)$, this violates relevance).

- $-\sigma_{3}$ is a relevant kernel contraction and a partial meet contraction.

- $-\gamma$ is a partial meet contraction and a relevant kernel contraction.

\section{Conclusions and future works}

We found an axiomatic characterization for ensconcement-based contraction and we defined a new contraction operator (basic related-AGM). With these results we extended the maps among different kinds of base contraction functions.

Regarding the significance of the result, the purpose of this article was not to analyse if ensconcement-based contraction is a suitable kind of contraction or not. Our purpose was to provide more tools that allow to compare different kinds of base contraction functions. Up to now, there was not a way to compare the ensconcement-based contraction with other approaches, except by means of examples. The axiomatic characterization provides a good tool to understand the behaviour of these functions and a metric to compare with other axiomatizations/postulates. Finally, this article can be used as a first step to discover the axiomatic characterization of related works.

In the near future we intend to analyse the following open questions:

- How to use the relationship $A-\alpha=(\operatorname{Cn}(A) \div \alpha) \cap A$ for other contraction functions like Levi contraction, Semi-Contraction or Severe Withdrawal (for an overview of these functions see $[12,13,32])$.

- How to extend the results for belief bases of partial meet contraction and kernel contraction for supplementary postulates.

- How to elucidate the relation among different kinds of supplementary postulates for belief bases. 


\section{Acknowledgements}

We would like to thank the audience at the Dagstuhl Seminar of Belief Change in Rational Agents and at the Eleventh International Workshop on Non-Monotonic Reasoning (NMR06) for many helpful comments and stimulating suggestions. Thanks are also due to the anonymous referees of NMR2006 and JLC for their numerous perceptive remarks and criticisms; and thanks to José Castanheira da Costa for corrections concerning presentation. M. R. is supported by FCT (Fundação para a Ciência e a Tecnologia).

\section{References}

[1] C. Alchourrón, P. Gärdenfors, and D. Makinson. On the logic of theory change: partial meet contraction and revision functions. Journal of Symbolic Logic, 50, 510-530, 1985.

[2] C. Alchourrón and D. Makinson. Hierarchies of regulations and their logic. In New Studies in Deontic Logic: Norms, Actions, and the Foundations of Ethics, R. Hilpinen, ed., pp. 125-148. D. Reidel Publishing Company, 1981.

[3] C. Alchourrón and D. Makinson. On the logic of theory change: Contraction functions and their associated revision functions. Theoria, 48, 14-37, 1982.

[4] C. Alchourrón and D. Makinson. On the logic of theory change: Safe contraction. Studia Logica, 44, 405-422, 1985.

[5] N. Belnap. Rescher's hypothetical reasoning: an amendment. In The philosophy of Nicholas Rescher:Discussion and Replies, E. Sosa, ed., pp. 19-28. D. Reidel, Dordrecht, 1979.

[6] M. Dalal. Investigations into a theory of knowledge base revision: Preliminary report. In Seventh National Conference on Artificial Intelligence, (AAAI-88), pp. 475-479. St. Paul, 1988.

[7] D. Dubois and H. Prade. Belief change and possibilistic logic. In Belief Revision, P. Gärdenfors, ed., number 29 in Cambridge Tracts in Theoretical Computer Science, pp. 142-182. Cambridge University Press, 1992.

[8] E. Fermé. Actualización de bases de conocimiento usando teorías de cambio de creencia. Iberamia, 92, 419-436, 1992.

[9] E. Fermé. Five faces of recovery. In Frontiers in Belief Revision, M.-A. Williams and H. Rott, eds, Applied Logic Series, pp. 247-259. Kluwer Academic Publishers, Dordrecht, The Netherlands, 2001.

[10] M. Falappa, E. Fermé, and G. Kern-Isberner. On the logic of theory change: relations between incision and selection functions. In Proceedings 17th European Conference on Artificial Intelligence, ECAI06, G. Brewka, S. Coradeschi, A. Perini, and P. Traverso, eds, pp. 402-406. IOS Press, Netherlands, 2006.

[11] A. Fuhrmann and S.O. Hansson. A survey of multiple contraction. Journal of Logic, Language and Information, 3, 39-74, 1994.

[12] E. Fermé and R. Rodríguez. A brief note about the Rott contraction. Logic Journal of the IGPL, 6, 835-842, 1998.

[13] E. Fermé and R. Rodríguez. Semi-contraction: Axioms and construction. Notre Dame Journal of Formal Logic, 39, 332-345, 1998.

[14] E. Fermé and H. Rott. Revision by comparison. Artificial Intelligence, 157, 5-47, 2004.

[15] A. Fuhrmann. Theory contraction through base contraction. Journal of Philosophical Logic, 20, 175-203, 1991.

[16] P. Gärdenfors. Rules for rational changes of belief. In Philosophical Essays dedicated to Lennart Aqvist on his fiftieth birthday, T. Pauli, ed., number 34 in Philosophical Studies, pp. 88-101, 1982. 
[17] P. Gärdenfors. Knowledge in Flux: Modeling the Dynamics of Epistemic States. The MIT Press, Cambridge, 1988.

[18] P. Gärdenfors and D. Makinson. Revisions of knowledge systems using epistemic entrench ment. In Proceedings of the Second Conference on Theoretical Aspects of Reasoning About Knowledge, M. Y. Vardi, ed., pp. 83-95, Morgan Kaufmann, Los Altos, 1988.

[19] A. Grove. Two modellings for theory change. Journal of Philosophical Logic, 17, 157-170, 1988.

[20] S. O. Hansson. Belief Base Dynamics. PhD thesis, Uppsala University, 1991.

[21] S. O. Hansson. A dyadic representation of belief. In Belief Revision, number 29 in Cambridge Tracts in Theoretical Computer Science, P. Gärdenfors, ed., pp. 89-121. Cambridge University Press, 1992.

[22] S. O. Hansson. In defense of base contraction. Synthese, 91, 239-245, 1992.

[23] S. O. Hansson. Kernel contraction. Journal of Symbolic Logic, 59, 845-859, 1994.

[24] S. O. Hansson. Taking belief bases seriously. In Logic and Philosophy of Science in Uppsala, Prawitz and Westerståhl, eds, pp. 13-28. Kluwer Academic Publishers, Dordrecht, 1994.

[25] S. O. Hansson. A survey of non-prioritized belief revision. Erkenntnis, 50, 413-427, 1999.

[26] S. O. Hansson. A Textbook of Belief Dynamics. Theory Change and Database Updating. Applied Logic Series. Kluwer Academic Publishers, Dordrecht, 1999.

[27] S. O. Hansson and Renata Wassermann. Local change. Studia Logica, 70, 49-76, 2002.

[28] B. Nebel. A knowledge level analysis of belief revision. In Proceedings of the 1st International Conference of Principles of Knowledge Representation and Reasoning, pp. 301-311. Morgan Kaufmann Publishers Inc., San Francisco, CA, USA, 1989.

[29] B. Nebel. How hard is it to revise a belief base? In Handbook of Defeasible Reasoning and Uncertainty Management Systems, Volume 3: Belief Change, D. Dubois and H. Prade, eds, pp. 77-145. Kluwer Academic Publishers, Dordrecht, 1998.

[30] H. Rott. Two methods of constructing contractions and revisions of knowledge systems. Journal of Philosophical Logic, 20, 149-173, 1991.

[31] H. Rott. "Just because". Taking belief bases seriously. In Logic Colloquium '98 - Proceedings of the Annual European Summer Meeting of the Association for Symbolic Logic. Lecture Notes in Logic, S. Buss, P. Hajek, and P. Pudlak, eds, vol. 13. Prague, Association for Symbolic Logic, 2000.

[32] H. Rott and M. Pagnucco. Severe withdrawal (and recovery). Journal of Philosophical Logic, 28, 501-547, 1999.

[33] W. Spohn. Ordinal conditional functions: A dynamic theory of epistemic states. In Causation in Decision, Belief Change and Statistics, W. Harper and B. Skyrms, eds, vol. 2, pp. 105-134. D. Reidel, Dordrecht, 1987.

[34] R. Wassermann. Resource Bounded Belief Revision. PhD thesis, University of Amsterdam, 2000.

[35] M.-A. Williams. Two operators for theory bases. In Proceedings Australian Joint Artificial Intelligence Conference, pp. 259-265. World Scientific, Hobart, Tasmania, 1992.

[36] M.-A. Williams. On the logic of theory base change. In Logics in Artificial Intelligence, C. MacNish, D. Pearce, and L. M. Pereira, eds, number 838 in Lecture Notes Series in Computer Science, pp. 86-105. Springer-Verlag, London, UK, 1994.

[37] M.-A. Williams. Transmutations of knowledge systems. In Proceedings of the fourth International Conference on Principles of Knowledge Representation and Reasoning, J. Doyle, E. Sandewall, and P. Torasso, eds, pp. 619-629. Morgan Kaufmann, Bonn, Germany, May 1994.

[38] M.-A. Williams. Iterated theory base change: A computational model. In Proceedings of the 14th IJCAI, pp. 1541-1547. Morgan Kaufmann Publishers, San Francisco, 1995. 


\section{Appendix: Proofs}

\section{A.1 Proof of Lemma 11}

(a), (b), (c) and (d) follow trivially from the definition of cut. (e) follows trivially from (d). If $\vdash \beta$, then (f)-(k) follow trivially from (a) and (e). Let $\nvdash \beta$ :

(f) Let $\alpha \preceq \beta$ and $\delta \in \operatorname{cut}_{A}(\beta)$. $\alpha \preceq \beta$ implies that $\alpha, \beta \in A$. We have to show that $\delta \in \operatorname{cut}_{A}(\alpha)$. Since $\delta \in \operatorname{cut}_{A}(\beta)$, from the definition of cut it follows that $\beta \prec \delta$. Then, by transitivity, we obtain $\alpha \prec \delta$, hence $\delta \in \operatorname{cut}_{A}(\alpha)$.

(g) Let $\alpha \prec \beta$. According to the definition of $c u t, \beta \in$ cut $_{A}(\alpha)$. Hence cut $_{A}(\alpha) \vdash \beta$. To conclude that $\operatorname{cut}_{A}(\beta) \nvdash \alpha$ it is enough to notice that, from $(f)$ we have $\operatorname{cut}_{A}(\beta) \subseteq \operatorname{cut}_{A}(\alpha)$, and that $(b)$ gives us that $\operatorname{cut}_{A}(\alpha) \nvdash \alpha$.

(h) By previous (d) it follows that $\operatorname{cut}_{A}(\alpha) \subseteq \operatorname{cut}_{A}(\alpha \wedge \beta)$. For the other direction, let $\alpha \prec \beta$ and $\delta \in A$ such that $\delta \notin \operatorname{cut}_{A}(\alpha)$. Due to (f) it follows that $\delta \notin c u t_{A}(\beta)$. Then, according to the definition of cut, $\{\mu \in A: \delta \preceq \mu\} \vdash \alpha$ and $\{\mu \in A: \delta \preceq \mu\} \vdash \beta$, from which it follows that $\{\mu \in A: \delta \preceq \mu\} \vdash \alpha \wedge \beta$. Hence $\delta \notin$ cut $_{A}(\alpha \wedge \beta)$.

(i) From (d) we obtain $\operatorname{cut}_{A}(\alpha) \subseteq \operatorname{cut}_{A}(\alpha \wedge \beta)$ and $\operatorname{cut}_{A}(\beta) \subseteq c u t_{A}(\alpha \wedge \beta)$. Let $\beta=\preceq \alpha$. cut $t_{A}(\alpha)=$ $\operatorname{cut}_{A}(\beta)$ follows immediately from (f). By the same arguments used in (h) we can prove that for $\delta \in A$ such that $\delta \notin c u t_{A}(\alpha)$ it follows that $\delta \notin c u t_{A}(\alpha \wedge \beta)$.

(j) Let $\mathrm{cut}_{A}(\alpha \wedge \beta) \neq \operatorname{cut}_{A}(\alpha)$. Due to (d) it follows that $\operatorname{cut}_{A}(\alpha) \subset c u t_{A}(\alpha \wedge \beta)$. Let $\delta \in c u t_{A}(\alpha \wedge$ $\beta) \backslash \operatorname{cut}_{A}(\alpha)$. Then $\{\gamma \in A: \delta \preceq \gamma\} \nvdash \alpha \wedge \beta$ and $\{\gamma \in A: \delta \preceq \gamma\} \vdash \alpha$. For all $\epsilon \in \operatorname{cut}_{A}(\alpha)$ it follows that $\{\mu \in A: \epsilon \preceq \mu\} \forall \alpha$. Then $\operatorname{cut}_{A}(\alpha) \subseteq\{\gamma \in A: \delta \preceq \gamma\}$. Then, since $\{\gamma \in A: \delta \preceq \gamma\} \forall \beta$ we can conclude that $\operatorname{cut}_{A}(\alpha) \forall \beta$.

(k) Let $\operatorname{cut}_{A}(\alpha \wedge \beta) \neq \operatorname{cut}_{A}(\beta)$. Due to (d) it follows that $\operatorname{cut}_{A}(\beta) \subset \operatorname{cut}_{A}(\alpha \wedge \beta)$. Let $\delta \in c u t_{A}(\alpha \wedge$ $\beta) \backslash \operatorname{cut}_{A}(\beta)$. Then $\{\gamma \in A: \delta \preceq \gamma\} \forall \alpha \wedge \beta$ and $\{\gamma \in A: \delta \preceq \gamma\} \vdash \beta$. Then $\{\gamma \in A: \delta \preceq \gamma\} \forall \alpha$, from which it follows that $\{\gamma \in A: \delta \preceq \gamma\} \subseteq \operatorname{cut}_{A}(\alpha)$. Hence $\operatorname{cut}_{A}(\alpha) \vdash \beta$.

\section{A.2 Proof of Theorem 14}

From Ensconcement-based Contraction to Postulates:

Success Let $\nvdash \alpha$ and assume by reductio that $A-\alpha \vdash \alpha$. Then it follows by compactness that there exists a finite subset of $A-\alpha, A^{\prime}=\left\{\beta_{1}, \ldots, \beta_{k}\right\}$, such that $A^{\prime} \vdash \alpha$. Then it follows from the definition of - that $\mathrm{cut}_{A}(\alpha) \vdash \alpha \vee \beta_{i}, i=1, \ldots, k$. Then $\operatorname{cut}_{A}(\alpha) \vdash \alpha \vee\left(\beta_{1} \wedge \beta_{2} \wedge \ldots \wedge \beta_{k}\right) \vdash \alpha$. Hence $c u t_{A}(\alpha) \vdash \alpha$. Contradiction by Lemma 11 (b).

Inclusion Trivial.

Vacuity Let $A \nvdash \alpha$ and let $\beta \in A$. By Lemma 11(c) it follows that $\operatorname{cut}_{A}(\alpha)=A$, from which it follows that $\operatorname{cut}_{A}(\alpha) \vdash \alpha \vee \beta$, hence, according to the definition of,$- \beta \in A-\alpha$.

Extensionality Let $\vdash \alpha \leftrightarrow \beta$. Then $\operatorname{cut}_{A}(\alpha)=\operatorname{cut}_{A}(\beta)$, and the rest follows trivially.

Disjunctive Elimination Let $\beta \in A$ and $\beta \notin A-\alpha$. Then it follows from the definition of - that $\operatorname{cut}_{A}(\alpha) \nvdash \alpha \vee \beta$. Assume by reductio that $A-\alpha \vdash \alpha \vee \beta$. Then compactness yields that there exists a finite subset of $A-\alpha, A^{\prime}=\left\{\beta_{1}, \ldots, \beta_{k}\right\}$, such that $A^{\prime} \vdash \alpha \vee \beta$. It follows from the definition of - that $\operatorname{cut}_{A}(\alpha) \vdash \alpha \vee \beta_{i}, \quad i=1, \ldots, k$. Then $\operatorname{cut}_{A}(\alpha) \vdash \alpha \vee\left(\beta_{1} \wedge \beta_{2} \wedge \ldots \wedge \beta_{k}\right) \vdash \alpha \vee \beta$. Hence $\operatorname{cut}_{A}(\alpha) \vdash \alpha \vee \beta$. Contradiction. 
Conjunctive Factoring If $\vdash \alpha \wedge \beta$ trivial from the definition of - . Let $\nvdash \alpha \wedge \beta$. We will prove by cases:

1. $\operatorname{cut}_{A}(\alpha) \vdash \beta$ : Then by Lemma 11(j) $\operatorname{cut}_{A}(\alpha \wedge \beta)=\operatorname{cut}_{A}(\alpha)$. We will prove by double inclusion that $A-\alpha \wedge \beta=A-\alpha$. Let $\gamma \in A-\alpha \wedge \beta$. It follows from the definition of - that $\gamma \in A$ and cut $_{A}(\alpha \wedge$ $\beta) \vdash(\alpha \wedge \beta) \vee \gamma$, then cut $_{A}(\alpha \wedge \beta) \vdash \alpha \vee \gamma$. Hence cut $_{A}(\alpha) \vdash \alpha \vee \gamma$, from which we can conclude that $\gamma \in A-\alpha$.

For the other inclusion, let $\gamma \in A-\alpha$. Then it follows from the definition of - that $\gamma \in A$ and cut $_{A}(\alpha) \vdash \alpha \vee \gamma$. Then cut $_{A}(\alpha \wedge \beta) \vdash \alpha \vee \gamma$. cut $t_{A}(\alpha) \vdash \beta$ yields cut $_{A}(\alpha) \vdash \beta \vee \gamma$, then cut $_{A}(\alpha \wedge \beta) \vdash \beta \vee$ $\gamma$. Hence $\operatorname{cut}_{A}(\alpha \wedge \beta) \vdash(\alpha \wedge \beta) \vee \gamma$. From the definition we can conclude that $\gamma \in A-\alpha \wedge \beta$.

2. $\operatorname{cut}_{A}(\beta) \vdash \alpha$ : Due to the symmetry of the case, it follows that $A-\alpha \wedge \beta=A-\beta$.

3. $\operatorname{cut}_{A}(\alpha) \nvdash \beta$ and $\operatorname{cut}_{A}(\beta) \nvdash \alpha$ : It follows by Lemma $11(\mathrm{k})$ that $\operatorname{cut}_{A}(\alpha \wedge \beta)=\operatorname{cut}_{A}(\alpha)=\operatorname{cut}_{A}(\beta)$. Let $\gamma \in A-\alpha \wedge \beta$. According to the definition of,$- \gamma \in A$ and cut $_{A}(\alpha \wedge \beta) \vdash(\alpha \wedge \beta) \vee \gamma$ iff cut $_{A}(\alpha \wedge$ $\beta) \vdash \alpha \vee \gamma$ and $\operatorname{cut}_{A}(\alpha \wedge \beta) \vdash \beta \vee \gamma$ iff $c u t_{A}(\alpha) \vdash \alpha \vee \gamma$ and $\operatorname{cut}_{A}(\beta) \vdash \beta \vee \gamma$ iff $\gamma \in A-\alpha$ and $\gamma \in A-\beta$.

\section{From Postulates to Ensconcement-based contraction:}

Let - be an operator to $A$ that satisfies success, inclusion, vacuity, extensionality, disjunctive elimination and conjunctive factoring. In order to prove that - is an ensconcement-based contraction we must prove that there exists a relationship $\preceq$ such that $\preceq$ satisfies $(\preceq \mathbf{1})-(\preceq \mathbf{3})$ and such that

(ebc) $A-\alpha= \begin{cases}\left\{\beta \in A: \text { cut }_{A}(\alpha) \vdash \alpha \vee \beta\right\} & \text { if } \forall \forall \alpha \\ A & \text { otherwise }\end{cases}$

where cut is defined in terms of $\preceq$.

Let $\preceq$ be defined as follows:

$$
\alpha \preceq \beta \text { iff }\left\{\begin{array}{l}
A-\alpha \wedge \beta \nvdash \alpha \\
o r \\
\vdash \alpha \wedge \beta
\end{array}\right.
$$

( $\preceq \mathbf{1})$ Let $\gamma \in A \backslash C n(\emptyset)$ and assume by reductio that $H=\{\alpha \in A: \gamma \prec \alpha\} \vdash \gamma$. Using our construction $\gamma \prec \alpha$ means that $A-\alpha \wedge \gamma \vdash \alpha, \forall \alpha \wedge \gamma$ and $A-\alpha \wedge \gamma \forall \gamma . \forall \alpha \wedge \gamma$ is redundant, then $H=\{\alpha \in A$ : $A-\alpha \wedge \gamma \vdash \alpha$ and $A-\alpha \wedge \gamma \forall \gamma\}$. It follows by conjunctive factoring that $A-\alpha_{i} \wedge \gamma=A-\gamma$ for each $\alpha_{i} \in H$. Then $H=\{\alpha \in A: A-\gamma \vdash \alpha$ and $A-\gamma \not \vdash \gamma\}$. Hence by success, inclusion and relative closure $H=A-\gamma$. Success contradicts $H \vdash \gamma$, since $A-\gamma \nvdash \gamma$.

( $\preceq 2$ ) Let $\nvdash \alpha$ and $\vdash \beta$. Due to success it follows that $A-\alpha \wedge \beta \forall \alpha \wedge \beta$, then $A-\alpha \wedge \beta \forall \alpha$. Then $\alpha \preceq \beta$. Since $\vdash \beta$, then $A-\alpha \wedge \beta \vdash \beta$ and due to $\nvdash \alpha \wedge \beta$ it follows that $\beta \npreceq \alpha$. Hence $\alpha \prec \beta$.

( $\preceq$ 3) Let $\alpha, \beta \in A$. Let $\vdash \alpha$ and $\vdash \beta$. Then $\vdash \alpha \wedge \beta$. Hence $\alpha \preceq \beta$.

(ebc) We will prove by cases:

1. $\vdash \alpha$. Follows trivially by failure.

2. $\nvdash \alpha$

$2.1 A \nvdash \alpha$. Vacuity yields $A-\alpha=A$. On the other hand (by Lemma 11 (c)) $c u t_{A}(\alpha)=A$, from which it follows that $\left\{\beta \in A: \operatorname{cut}_{A}(\alpha) \vdash \alpha \vee \beta\right\}=A$.

$2.2 A \vdash \alpha$.

2.2.1 $\alpha \in A$. We will first replace $\prec$ in $\operatorname{cut}_{A}(\alpha)$ by our construction:

Due to Lemma 10

$$
\begin{aligned}
& \operatorname{cut}_{A}(\alpha)=\{\delta \in A: \alpha \prec \delta\} \\
& \operatorname{cut}_{A}(\alpha)=\{\delta \in A: \alpha \preceq \delta \text { and } \delta \npreceq \alpha\} \\
& \operatorname{cut}_{A}(\alpha)=\{\delta \in A: A-\alpha \wedge \delta \nvdash \alpha \text { and } A-\alpha \wedge \delta \vdash \delta\}
\end{aligned}
$$

it follows by conjunctive factoring that $A-\alpha \wedge \delta=A-\alpha$. Then (since, by success, $A-\alpha \nvdash \alpha$ )

$$
\operatorname{cut}_{A}(\alpha)=\{\delta \in A: A-\alpha \vdash \delta\},
$$


hence by relative closure and inclusion,

Replacing in (ebc):

$$
\operatorname{cut}_{A}(\alpha)=A-\alpha
$$

$$
\beta \in A-\alpha \text { iff } \beta \in A \text { and } A-\alpha \vdash \alpha \vee \beta,
$$

that trivially follows from inclusion and disjunctive elimination.

2.2.2 $\alpha \notin A$. First, we will construct $A^{\prime}=A \cup\{\alpha\}$ and let $\preceq^{\prime}$ such that for all $\beta, \gamma$ in $A, \beta \preceq^{\prime} \gamma$ iff $\beta \preceq \gamma, \alpha \prec^{\prime} \beta$ if $\operatorname{cut}_{A}(\alpha \wedge \beta) \neq \operatorname{cut}_{A}(\beta), \beta \prec^{\prime} \alpha$ if $\operatorname{cut}_{A}(\alpha \wedge \beta) \neq \operatorname{cut}_{A}(\alpha)$ and $\alpha=\preceq \beta$ if $\operatorname{cut}_{A}(\alpha \wedge \beta)=$ cut $_{A}(\alpha)=\operatorname{cut}_{A}(\beta)$. Let cut $_{A^{\prime}}^{\prime}(\alpha)$ be defined in terms of $A^{\prime}$ and $\preceq^{\prime}$. Due to the previous proof 2.2.1, we will prove this case by proving that $\left\{\beta \in A:\right.$ cut $\left._{A}(\alpha) \vdash \alpha \vee \beta\right\}=\left\{\beta \in A^{\prime}: c u t_{A^{\prime}}^{\prime}(\alpha) \vdash \alpha \vee \beta\right\}$. To do that we must prove (a) $\preceq^{\prime}$ is an ensconcement and (b) $\operatorname{Cn}\left(\operatorname{cut}_{A}(\beta)\right)=\operatorname{Cn}\left(\operatorname{cut}_{A^{\prime}}^{\prime}(\beta)\right)$. Part (a) is trivial. For part (b) we have that $\mathrm{cut}_{A^{\prime}}^{\prime}(\beta)=\left\{\delta \in A^{\prime}:\left\{\gamma \in A^{\prime}: \delta \preceq^{\prime} \gamma\right\} \forall \beta\right\}=\{\delta \in A \cup\{\alpha\}:\{\gamma \in A \cup\{\alpha\}: \delta \preceq \gamma\} \forall \beta\}$. If $\alpha \notin$ cut $_{A^{\prime}}^{\prime}(\beta)$ it follows that cut $_{A^{\prime}}^{\prime}(\beta)=\operatorname{cut}_{A}(\beta)$. If $\alpha \in \operatorname{cut}_{A^{\prime}}^{\prime}(\beta)$ it follows that $\left\{\gamma \in A^{\prime}: \alpha \preceq \gamma\right\} \nvdash \beta$, from which it follows that $\alpha \npreceq \beta$. Then $\beta \prec \alpha$, hence (by Lemma $11(\mathrm{~g})) \operatorname{cut}_{A}(\beta) \vdash \alpha$, from which it follows that $\operatorname{Cn}\left(\operatorname{cut}_{A}(\beta)\right)=\operatorname{Cn}\left(\operatorname{cut}_{A^{\prime}}^{\prime}(\beta)\right)$.

\section{A.3 Proof of Observation 15}

Counterexample: Let $A=\{\alpha \wedge \beta, \alpha \vee \gamma, \gamma\}$ and let $\preceq$ be an ensconcement on $A$ such that $\gamma \prec \alpha \wedge$ $\beta \prec \alpha \vee \gamma$. Then $\operatorname{cut}_{A}(\alpha)=\operatorname{cut}_{A}(\beta)=\{\alpha \vee \gamma\} . \gamma \in A-\alpha$, since $\operatorname{cut}_{A}(\alpha) \vdash \alpha \vee \gamma$, but $\gamma \notin A-\beta$, since $\operatorname{cut}_{A}(\beta) \nvdash \beta \vee \gamma$. Hence uniformity fails.

\section{A.4 Proof of Observation 16}

Let $\beta \in A$ and $\beta \notin A-\alpha$. Then by relevance there is some set $A^{\prime}$ such that $A-\alpha \subseteq A^{\prime} \subseteq A$ and $\alpha \notin$ $\operatorname{Cn}\left(A^{\prime}\right)$ but $\alpha \in \operatorname{Cn}\left(A^{\prime} \cup\{\beta\}\right)$, from which it follows by deduction that $\beta \rightarrow \alpha \in \operatorname{Cn}\left(A^{\prime}\right)$. Hence (due to $\left.\alpha \notin C n\left(A^{\prime}\right)\right), \alpha \vee \beta \notin C n\left(A^{\prime}\right)$.

\section{A.5 Proof of Observation 18}

Counterexample: (Assume that core-retainment and disjunctive elimination are satisfied for all $\delta \neq$ $\alpha)$. Let $A=\{\beta, \gamma \rightarrow \alpha, \gamma \wedge(\beta \rightarrow \alpha)\}$. Let $A-\alpha=\{\gamma \rightarrow \alpha\}$. Disjunctive elimination is satisfied since $A-\alpha \nvdash \alpha \vee \beta$ and $A-\alpha \nvdash \alpha \vee(\gamma \wedge(\beta \rightarrow \alpha))$. To show that core-retainment is satisfied for $\beta$ let $A^{\prime}=$ $\{\gamma \wedge(\beta \rightarrow \alpha)\}$ and for $\gamma \wedge(\beta \rightarrow \alpha)$ let $A^{\prime}=\{\gamma \rightarrow \alpha\}$. However, relevance fails for $\beta$.

\section{A.6 Proof of Observation 19}

Let $\beta \in A$ and $\beta \notin A-\alpha$. Then it follows by disjunctive elimination that $A-\alpha \nvdash \alpha \vee \beta$. By deduction $(A-\alpha)+\neg \beta \nvdash \alpha$, from which it follows that $(A-\alpha)+\neg \beta \vee \alpha \nvdash \alpha$. Let $B=(A-\alpha)+\neg \beta \vee \alpha$. Due to $\beta \in A$ and $\beta \notin A-\alpha$ vacuity yields that $A \vdash \alpha$. From inclusion $A-\alpha \subseteq A$. Hence (by monotony) we have $B \subseteq A+\neg \beta \vee \alpha=A$. Since $B \nvdash \alpha$, and $B+\beta \vdash \alpha$, we conclude that - satisfies relevance.

\section{A.7 Proof of Observation 21}

(For this proof we assume that the reader is familiar with the AGM contraction functions).

$(\Leftarrow)$ Let $\div$ be an operator on $C n(A)$ that satisfies the basic AGM postulates (closure, success, vacuity, inclusion, recovery and, extensionality), and let - be such that $A-\alpha=\operatorname{Cn}(A) \div \alpha \cap A$. 
Success, inclusion, vacuity and extensionality follow trivially from the definition. For disjunctive elimination let $\beta \in A$ and $\beta \notin A-\alpha$. Then (by closure) $C n(A) \div \alpha \nvdash \beta$, from which it follows by recovery that $C n(A) \div \alpha \vdash \neg \alpha \vee \beta$. Then it follows by success and vacuity that $C n(A) \div \alpha \nvdash \alpha \vee \beta$. Hence $A-\alpha \nvdash \alpha \vee \beta$.

$(\Rightarrow)$ Let - be an operator on $A$ that satisfies success, inclusion, vacuity, extensionality and disjunctive elimination and let $\div$ defined as follows:

$$
C n(A) \div \alpha=C n\left(A-\alpha \cup\left(\bigcup_{\beta_{i} \in A} \alpha \rightarrow \beta_{i}\right)\right)
$$

We must prove: (i) that $\div$ is a basic AGM contraction function, (ii) that $\div$ satisfies $A-\alpha=C n(A) \div \alpha \cap A$.

(i) Closure follows trivially from the definition. Success and extensionality follow trivially from the definition and -success and - extensionality respectively. Vacuity follows from - vacuity and from $\left(\bigcup_{\beta_{i} \in A} \alpha \rightarrow \beta_{i}\right) \subseteq C n(A)$. Inclusion follows from -inclusion and from $\left(\bigcup_{\beta_{i} \in A} \alpha \rightarrow \beta_{i}\right) \subseteq C n(A)$. For recovery let $\gamma \in C n(A)$ and $\gamma \notin C n(A) \div \alpha$, we must prove that $\alpha \rightarrow \gamma \in C n(A) \div \alpha$ that follows trivially from our definition of $\div$.

(ii) We must prove that $A-\alpha=\operatorname{Cn}\left(A-\alpha \cup\left(\bigcup_{\beta_{i} \in A} \alpha \rightarrow \beta_{i}\right)\right) \cap A$. We will prove by double inclusion. Let $\gamma$ be a sentence. If $\vdash \gamma \rightarrow \alpha$, trivial by success. Let $\forall \gamma \rightarrow \alpha$. For one side let $\gamma \in A-\alpha$. Then by inclusion $\gamma \in A$ and the rest follows trivially. For the other side let $\gamma \in C n\left(A-\alpha \cup\left(\bigcup_{\beta_{i} \in A} \alpha \rightarrow \beta_{i}\right)\right) \cap A$. Then $\gamma \in A$ and $\gamma \in C n\left(A-\alpha \cup\left(\bigcup_{\beta_{i} \in A} \alpha \rightarrow \beta_{i}\right)\right.$ ) from which it follows (due to $\bigcup_{\beta_{i} \in A} \alpha \rightarrow \beta_{i} \vdash \neg \alpha \vee \gamma$ and $\left.\bigcup_{\beta_{i} \in A} \alpha \rightarrow \beta_{i} \forall \alpha \vee \gamma\right)$ that $A-\alpha \vdash \alpha \vee \gamma$. Hence by disjunctive elimination $\gamma \in A-\alpha$.

Received December 2007 